Preprints of the

Max Planck Institute for

Research on Collective Goods

Bonn 2009/11

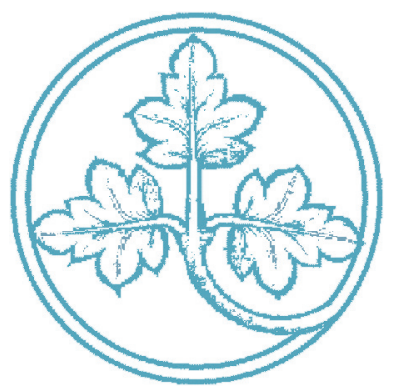

Persistence of Monopoly and Research Specialization

Philipp Weinschenk

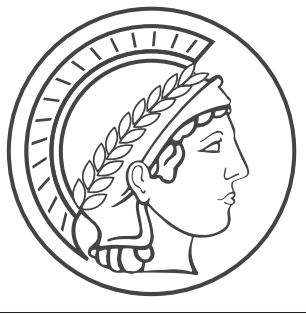




\section{Persistence of Monopoly and Research Specialization}

Philipp Weinschenk

April 2009 


\title{
PERSISTENCE OF MONOPOLY AND RESEARCH SPECIALIZATION
}

\author{
Philipp Weinschenk* \\ Bonn Graduate School of Economics \\ and Max Planck Institute for Research on Collective Goods
}

April 1, 2009

\begin{abstract}
We examine the persistence of monopolies in markets with innovations when the outcome of research is uncertain. We show that for low success probabilities of research, the incumbent can seldom preempt the potential entrant. Then the efficiency effect outweighs the replacement effect. It is vice versa for high probabilities. Moreover, the incumbent specializes in "safe" research and the potential entrant in "risky" research. We also show that the probability of entry has an inverted U-shape in the success probability. Since even at the peak entry is rather unlikely, the persistence of the monopoly is high.
\end{abstract}

Keywords: Persistence of Monopoly; Efficiency Effect; Replacement Effect; Stochastic Innovations.

JEL Classification: L12, O31.

\section{INTRODUCTION}

This paper takes a new look at monopoly persistence in markets with innovations. The extensive literature on the subject has discussed this issue in terms of the efficiency effect and the replacement effect. Since competition destroys profits, the efficiency effect predicts that the incumbent's incentive to remain a monopolist through innovating is at least as great as the entrant's incentive to become a duopolist. The replacement effect (Arrow, 1962) predicts the opposite: The entrant's incentive to innovate is higher than the incumbent's because only the incumbent takes into account that innovating replaces its existing technology. According

* Max Planck Institute for Research on Collective Goods, Kurt-Schumacher-Str. 10, 53113 Bonn, Germany, weinschenk@coll.mpg.de. I thank Philippe Aghion, Felix Bierbrauer, Stefanie Brilon, Andrew Daughety, Mathias Dewatripont, Christoph Engel, Paul Heidhues, Martin Hellwig, Fabian Herweg, Jos Jansen, Stephan Lauermann, Daniel Müller, Tymofiy Mylovanov, Jennifer Reinganum, Nora Szech, Andrea Wittke, as well as seminar participants at the Max Planck Institute for Research on Collective Goods, the 2007 Summer School on Contract Theory at Bronnbach, the University of Bonn, the 2008 ZEW Conference on the Economics of Innovation and Patenting at Mannheim, and the 2008 EEA Conference at Milan. 
to the classical work of Gilbert and Newbery (1982; henceforth GN) the efficiency effect determines the outcome, whereas in a seminal paper by Reinganum (1983; henceforth RE) it is the replacement effect. ${ }^{1}$ We build a unifying model in which both effects are present. We allow for uncertainty with respect to the outcomes of innovative activities and show that the success probability of research determines the relative strength of both effects.

We consider a monopolized market, where the first-moving incumbent may be able to discourage the potential entrant from investing in research by investing itself. ${ }^{2}$ The outcomes of research activities are uncertain. Therefore, preemption is less than perfect. For high success probabilities we obtain a result in the spirit of GN: preemption is almost perfect so the efficiency effect is the driving force. Intuitively, since the success probability is high the potential entrant's expected profit from research greatly depends on the incumbent's research decision. Hence, it is very likely that the incumbent can and does preempt the potential entrant. For low success probabilities the same argument applies in reverse, i.e., the incumbent can hardly preempt and so the replacement effect predominates. This result is in the spirit of RE.

These results imply that research with a high success probability is more likely done by the incumbent than by the potential entrant, and it is vice versa for research with a low success probability. In this sense, incumbents specialize in "safe" research, and potential entrants in "risky" research. We also show that research undertaken by potential entrants is, on average, "riskier" than that of incumbent firms. Moreover, the probability of entry has - at least roughly - an inverted U-shape in the success probability of research. Since even at the peak the probability of entry is only a quarter, the persistence of monopoly is high.

We also explore the normative aspects of our model. We consider the second best world in which pricing cannot be regulated and show that, apart from one exception, firms never overinvest and may underinvest. When the incumbent preempts the potential entrant and the innovation is non-drastic, overinvestment may occur. ${ }^{3}$ Intuitively, this holds when in case that incumbent's research is successful (i) the monopoly price is almost the same as when the incumbent would have the old technology (so

\footnotetext{
${ }^{1}$ See also the debate in Reinganum (1984) and Gilbert and Newbery (1984).

${ }^{2}$ The idea that a dominant firm might use its investment decision as a strategic device to persuade a potential entrant not to enter stems from Spence (1977) and Dixit (1980). They consider capacity investments.

${ }^{3} \mathrm{An}$ innovation is called drastic if it is so large that the innovative entrant is effectively unconstrained by incumbent's competition. It can charge monopoly prices and yield monopoly profits.
} 
that the consumer surplus is hardly increased) and (ii) the incumbent's profit only slightly improves relative to the investment costs.

The research process considered by GN is commonly interpreted as an auction. As an extension, we integrate such an auction process into our model. This changes our results: regardless of the success probability, the incumbent will always outbid the entrant if the innovation is nondrastic. So entry will never occur. This replicates GN's result in a more general framework which allows for uncertainty of the research process.

Our paper is related to the literature on the persistence of monopoly in markets with innovations, which is surveyed by Gilbert (2006). The relation of our model to GN and RE is discussed later. Denicolo (2001) and Etro (2004) consider a research process of the RE type where the replacement effect disappears, since the aggregate R\&D effort is independent of the incumbent's decision. Fudenberg and Tirole (1986) show that in RE's model, when the innovation is non-drastic, the efficiency effect may outweigh the replacement effect. With different outcomes being possible, there is, however, no clear-cut result. ${ }^{4}$ Our model delivers clear and intuitive results without any assumption on whether the innovation is drastic or not.

We offer a novel explanation to the question why entrants do riskier research than incumbents. Existing literature on this question emphasizes other explanations. While Kihlstrom and Laffont (1979) look at differences in the risk-attitudes of firms, De Meza and Southey (1996) consider excessive optimism of entrepreneurs. Scherer and Ross (1990, Ch. 17) blame the bureaucracy in large companies. Baumol (2004) highlights educational differences between researchers in incumbent firms and entrepreneurs that engage in research. In Rosen (1991) the ex ante highcost firm must spend more than the ex ante low-cost firm to yield the same cost level. Through this asymmetry, the former chooses a riskier research project than the latter.

We present, analyze, and discuss the model in Sections 2, 3, and 4, respectively. A welfare analysis is in Section 5. After considering an auction setting in Section 6, we conclude in Section 7. Proofs are in the Appendix.

${ }^{4}$ See Fudenberg and Tirole (1986, Ch. 3) and Tirole (1988, pp. 397-398). Also Beath, Katsoulacos, and Ulph (1989a, 1989b) show that both effects can play a role in a model similar to RE's. But again, no clear and simple results can be yielded (1989a, p. 167). 


\section{MODEL}

There are two firms, an incumbent $I$, and a potential entrant $E$. At stage 1 the incumbent decides whether or not to invest in a firm specific research project. Investing causes expected costs of $k>0$ and yields an innovation with probability $p \in(0,1]$. At stage 2 firm $E$ faces the same decision. In order to focus on the replacement and the efficiency effect we set both firms on equal footing and assume that both firms' projects have the same costs and success probabilities. At stage 3 nature independently determines whether each firm's project is successful or not. A successful firm gets a process innovation that enables production at per-unit costs of $\underline{c}$. If $I$ does not invest or its project fails, it can produce at per-unit costs of $\bar{c}$ by using its old technology, where $\bar{c}>\underline{c}>0$. In contrast, if $E$ does not invest or its project fails, it cannot produce at all. Finally, at stage 4, firms compete à la Bertrand. For reasons that will become clear later, we assume that there is also a stage 0 where first the success probability $p$ is drawn from density $g$, and then the cost $k$ is drawn from conditional density $h .^{5}$

Firms are risk neutral and cannot collude. There is perfect information. The solution concept is subgame perfect Nash equilibrium. Consumer demand is given by the function $D(\phi)$, where $\phi$ is the consumer price. We assume that $D(\phi)$ is falling in $\phi$, positive for $\phi=\bar{c}+\epsilon$ (where $\epsilon$ is small and positive), and $D(\underline{c})$ is finite. These assumptions allow us to borrow Tirole's (1988) analysis of Bertrand profits; see below. To yield clear-cut normative results we have to assume that monopolist's optimal price is unique. ${ }^{6}$

\section{ANALYSIS}

We solve the model by backward induction. In this section we first describe the Bertrand profits of firms. Then we determine their research decisions. Finally we present the results.

\subsection{BERTRAND PROFITS}

Bertrand profits are uniquely determined by the firms' production costs and therefore can be expressed as $\pi^{J}\left(c^{I}, c^{E}\right)$, where $J \in\{I, E\}, c^{I} \in$ $\{\bar{c}, \underline{c}\}$, and $c^{E} \in\{\underline{c},-\}$. The symbol "-" indicates that $E$ cannot produce

\footnotetext{
${ }^{5}$ When $k$ is drawn before or simultaneously to $p$, we can ignore the $k$ value until $p$ is drawn, and so preserve the vision that $p$ is drawn first.

${ }^{6}$ Hermalin (2009) offers some weak assumptions on $D(\cdot)$ that guarantee inter alia uniqueness; see his Proposition 3. The key assumption is that $D(\cdot)$ is $\log$-concave.
} 
at all. We normalize the maximal profit $\pi^{I}(\underline{c},-)$ to 1 . The following lemma on the structure of firms' profits is due to Tirole (1988). ${ }^{7}$

L E M M A 1 :

(i) $\pi^{E}(\bar{c},-)=\pi^{E}(\underline{c},-)=\pi^{E}(\underline{c}, \underline{c})=\pi^{I}(\underline{c}, \underline{c})=\pi^{I}(\bar{c}, \underline{c})=0$,

(ii) $1>\pi^{I}(\bar{c},-)>0$,

(iii) $1 \geq \pi^{E}(\bar{c}, \underline{c})>1-\pi^{I}(\bar{c},-)$.

Part (i) describes the well-known result that when a firm cannot produce or has the same or even higher per-unit costs than its competitor its Bertrand profit is zero. Part (ii) states that a monopolist is strictly better off with low than with high per-unit costs; nonetheless, a monopolist with high per-unit costs makes a positive profit. The first inequality of part (iii) contains the efficiency effect: since competition destroys industry profits, I's incentive to remain a monopolist through innovating [and yield a Bertrand profit of 1 ] is at least as great as $E$ 's incentive to become a duopolist [which yields a Bertrand profit of $\pi^{E}(\bar{c}, \underline{c})$ ]. However, when $I$ takes into account that its old technology is replaced when it innovates [the net-effect of innovating on its Bertrand profit is just $\left.1-\pi^{I}(\bar{c},-)\right] E$ 's incentive to innovate is higher than I's. This is the replacement effect which is captured by the last inequality. When the first weak inequality of (iii) is strict we say that the innovation is non-drastic; in case of equality the innovation is called drastic.

\subsection{RESEARCH DECISIONS}

Firm $J$ 's research decision, $J \in\{I, E\}$, is denoted by $a^{J} \in\{0,1\}$, where 0 denotes no investment and 1 investment. We assume that in case of indifference a firm does not invest. ${ }^{8}$

Potential Entrant's Research Decision. - Since $a^{I}$ is either 0 or 1 , there are two subgames. Letting $b^{E}\left(a^{I}\right)$ denote $E$ 's best responses to $a^{I}$, we have

$$
\begin{aligned}
& b^{E}[0]=1 \quad \Longleftrightarrow \quad k<\pi^{E}(\bar{c}, \underline{c}) p=: \bar{k}(p) ; \\
& b^{E}[1]=1 \quad \Longleftrightarrow \quad k<\pi^{E}(\bar{c}, \underline{c})(1-p) p=: \underline{k}(p) .
\end{aligned}
$$

Thus, $E$ invests if and only if the costs $k$ are sufficiently low. More specifically, if $k<\underline{k}(p)$, then $a^{E}=1$ is E's dominant strategy and when

\footnotetext{
${ }^{7}$ Tirole partially summarizes existing literature. He does not consider the Nash equilibria found by Blume (2003) where one firm plays a weakly dominated strategy; see Tirole (1988, p. 234, footnote 37$)$.

${ }^{8}$ To rule out that cases of indifference drive our results we will later introduce an assumption that guarantees that cases of indifference have a measure of zero.
} 
$k \geq \bar{k}(p)$ the dominant strategy is $a^{E}=0$. For $k \in[\underline{k}(p), \bar{k}(p))$ we have $b^{E}(0)=1$ and $b^{E}(1)=0$.

Incumbent's Research Decision. - $I$ chooses the optimal action foreseeing $E$ 's later responses. If $k \geq \bar{k}(p)$, then

$$
a^{I}=1 \Longleftrightarrow k<\left(1-\pi^{I}(\bar{c},-)\right) p=: \hat{k}(p)
$$

if $k<\underline{k}(p)$, then

$$
a^{I}=1 \Longleftrightarrow k<\left(1-\pi^{I}(\bar{c},-)\right)(1-p) p=: \tilde{k}(p) ;
$$

and if $k \in[\underline{k}(p), \bar{k}(p))$, then

$$
a^{I}=1 \Longleftrightarrow k<p
$$

Equilibrium. - Using the previous formulas the construction of the equilibria is straightforward. Figure 1 shows the equilibrium research decisions, which we denote by $a^{*}=\left(a^{I *}, a^{E *}\right)$. The following lemma summarizes formally.

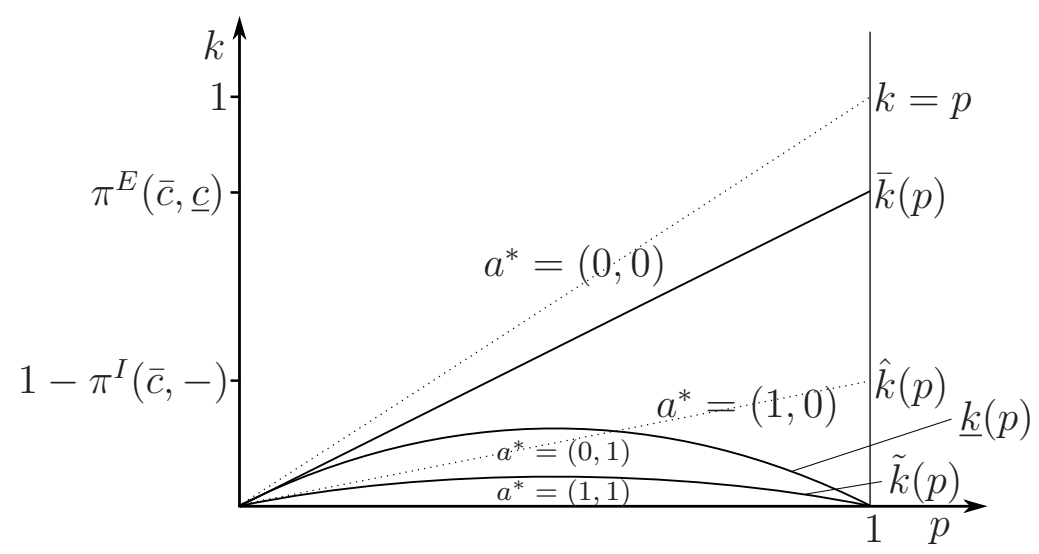

Figure 1: Research decisions in equilibrium.

L E M M A 2: The firms' research decision in equilibrium are

- $a^{*}=(1,1)$ for $k \in[0, \tilde{k}(p))$;

- $a^{*}=(0,1)$ for $k \in[\tilde{k}(p), \underline{k}(p))$;

- $a^{*}=(1,0)$ for $k \in[\underline{k}(p), \overline{\bar{k}}(p))$;

- $a^{*}=(0,0)$ for $k \geq \bar{k}(p)$.

Intuition. - Investments are strategic substitutes. In the parameter area where $k \in[\underline{k}(p), \bar{k}(p)) E$ invests if and only if $I$ has not invested. Hence, $I$ can preempt $E$. But is it profitable for $I$ to preempt? Yes it 
is, due to the efficiency effect: in this parameter area $k<p$, i.e., (5) holds. Roughly speaking, $I$ prefers the risk to replace its old technology itself to the risk being replaced by $E$. Hence in this parameter set the equilibrium is $a^{*}=(1,0)$. Note, in this parameter set regardless whether $I$ invests or not, exactly one firm will invest and so eventually make $I$ 's old technology obsolete. Consequently, $I$ does not take into account that its existing technology may be replaced by its own investment and the replacement effect plays no role.

For low $p$ values preemption is possible only for a relatively small range of costs, compared to the range of costs where at least one firm invests. The line of arguments is as follows. Observe that the probability that only $E$ receives the innovation when it invests - and thus receives a positive Bertrand profit - hardly changes through I's decision. Therefore, $E$ 's expected profit from research hardly depends on I's decision. Hence, preemption is seldom possible. In contrast, for high $p$ values, preemption is mostly possible; the arguments stated before apply in reverse. So research is a powerful preemption device if and only if it is likely to succeed.

Due to the replacement effect, there is a set of parameters where $E$ is willing to invest, irrespective of what $I$ has done, but where $I$ is no longer motivated to invest, given that $E$ will invest. Hence in this set $a^{*}=(0,1)$. As explained before, when the success probability $p$ is high, $E$ 's expected profit from research - and so its willingness to invest - is very sensitive upon I's investment decision. Hence the replacement effect loses its power when $p$ becomes large. In the extreme case of $p=1$, it has no power at all: $E$ never invests when $I$ has invested.

When the costs are very low both firms are always willing to invest and hence $a^{*}=(1,1)$. In the remaining parameter set, costs are so high that $a^{*}=(0,0)$.

\subsection{RESULTS}

From Figure 1 or the arguments made before it is intuitive that when the success probability $p$ is low the replacement effect is "more important" than the efficiency effect, whereas for high $p$ values it is vice versa. To formalize this intuition, we assume that prior to the firms' investment decisions the costs $k$ and the success probability $p$ are drawn. This is kind of a comparative statics analysis which allows us to determine how "important" the different equilibria and effects are.

In order to obtain concrete results we make the following assumption which says that $k$ is uniformly distributed in the "relevant" set. 
A S S U M P T I O N A 1: The conditional density of $k, h(k \mid p)$, is uniform in $k$ for $(p, k) \in \mathcal{S}:=\{(p, k) \mid 0<p \leq 1,0<k \leq \bar{k}(p)\}$.

With this assumption we can establish our main result which is about the probability of investments, conditional on $p$. Note, we take the perspective that $k$ is not yet drawn.

P R O P O S I T I O N 1: Suppose A1 holds. If $p$ is sufficiently high, $I$ is more likely to invest in research than $E$. It is vice versa if $p$ is sufficiently low.

Intuitively, given that a high $p$ value was drawn, it is much less likely that a $k$ will be drawn which lies in the small interval where $a^{*}=(0,1)$ rather than in the large interval where $a^{*}=(1,0)$; see Figure 1. For a low $p$ value it is vice versa. Put differently, when the success probability is high it is likely that the incumbent preempts the potential entrant and so that the efficiency effect determines the outcome. This is not true when the success probability is low. Then the replacement effect predominates. So both effects are important in our model, and the success probability determines their relative power.

Taking another view by looking at a large number of $I-E$-pairs, Proposition 1 predicts that most of the "risky" research is done by potential entrants but not by incumbents. Incumbents on the other hand specialize in "safe" research, which is undertaken rarely by potential entrants. ${ }^{9}$

Perhaps not surprising - albeit nontrivial to prove - is that potential entrants' research is "riskier" than that of the incumbent. We measure the likelihood of failure when neither $p$ nor $k$ is yet drawn. Additionally to A1 we assume that $p$ is distributed with positive and non-atomic density.

P R O P O S I T I O N 2: Suppose A1 holds and that $g(p)$ has full support and is finite $\forall p \in(0,1]$. Then $E$ 's research is more likely to fail than I's.

The sketch of the proof is as follows: First we show that the likelihood that $I$ invests relative to the likelihood that $E$ invests is increasing in $p$. Then we show that this implies that the distribution of $p$, conditional that $I$ invests, first-order stochastically dominates the distribution of $p$, conditional that $E$ invests. This enables us to finally prove that $E$ 's research is more likely to fail than $I$ 's.

What remains to determine is the persistence of monopoly. When $E$ has invested and is successful, it competes with $I$ on the market. Then

\footnotetext{
${ }^{9}$ We say that research is "risky" ("safe") when $p$ is low (high). This denomination is cheeky because when $p$ is low the outcome of the research process can be less uncertain than when $p$ is high.
} 
the monopoly does not persist. Otherwise the monopoly persists. We can deliver the following result about the probability of entry (i.e., nonpersistence of the monopoly), conditional on $p$.

P R O P O S I T I N 3: Suppose A1 holds. Then the probability of entry is at most $p(1-p)$.

The intuition is simple: When $p$ is low, research is seldom successful and entry rarely occurs. When $p$ is high, on the other hand, $I$ preempts $E$ except for a small interval of cost realizations; see Proposition 1. Therefore entry is unlikely, too. For intermediate values of $p$, however, it is likely that $E$ invests and is successful. Hence, the probability of entry has roughly an inverted U-shape in $p .{ }^{10}$ By Proposition 3, the probability of entry is at most $1 / 4$, which implies that the probability for the monopoly to persist is at least $3 / 4 .^{11}$ Hence we conclude that the persistence of the monopoly is high.

\section{DISCUSSION}

\subsection{EMPIRICAL EVIDENCE}

Baumol (2004) finds that "risky" research is most often done by entrepreneurs and not by incumbents, and that it is vice versa for "safe" research. ${ }^{12}$ This fits Proposition 1. Also Vinod Koshla notes that "[r]isk and acceptance of failure are central to innovation, [...] but the dinosaurs typically avoid both" (Statement in The Economist, 2007, p. 3). For industries in which failures are common, our model predicts that most of the research is done by potential entrants and not by incumbents. This seems to be the case, for example, in the IT sector.

Proposition 2, which states that E's research is more likely to fail than I's, is supported by Astebo's (2003, p. 227) finding that

"the average probability that an independent inventor succeeds in commercialising his/her invention is estimated to about 0.07 . In comparison, the probability of commercial success of conducting R\&D in established firms is approximately $0.27, "$

\footnotetext{
${ }^{10}$ Ignoring the probability mass outside $\mathcal{S}$ (this is possible when one redefines the densities $g$ and $h$ accordingly) yields an exact inverted U-shape.

${ }^{11}$ The reason why the persistence is not lower in our model when the replacement effect is powerful is that the replacement effect is only strong when research often fails - and failure of potential entrant's research is another reason for persistence.

${ }^{12}$ See also Scherer and Ross (1990, p. 653).
} 
where the later value is from Mansfield et al. (1977). Further evidence is provided by Baumol (2004) and Bianchi and Henrekson (2005, p. 367).

Empirically, the persistence of monopolies seems to be high (Geroski, 1995), as Proposition 3 predicts.

\subsection{THE ROLE OF ASSUMPTION A1}

Scherer and Ross (1990) elucidate how the costs of R\&D are distributed. However, since we use expected costs and normalize them we cannot use their insights. To us, A1 seems a natural starting point. This assumption is not as restrictive as it may seem for several reasons. First, A1 assumes that $h(\cdot)$ is uniform in $k$ for parameters of the set $\mathcal{S}$, but $h(\cdot)$ may still depend upon $p$. Second, when the average density of $k$ conditional on $p$ in the different equilibrium sets of $\mathcal{S}$ is the same, the proofs, and so also our results, stay unchanged. ${ }^{13}$ Third, A1 is sufficient, but not necessary, for our results.

To see the last point, suppose that A1 does not hold. Proposition 1 also holds under the alternative, weak assumption: the average density of $k$, conditional on $p$ and that either only $I$ or $E$ invests, is bounded between two positive constants. ${ }^{14}$ Additionally observe that then the probability of entry approaches 0 , as $p$ approaches 0 or 1 . So at least roughly the probability of entry has an inverted U-shape in the success probability of research; cf. Proposition 3.

A1 is important for Proposition 2. When A1 does not hold, the likelihood that $I$ invests relative to the likelihood that $E$ invests can be locally decreasing in $p$. Hence, the result stated in Proposition 2 can reverse. From Figure 1 it is, however, intuitive that for "many distributions" of $k$ and $p$ the result holds. ${ }^{15}$

\footnotetext{
${ }^{13}$ More technically this means that for all $p \in(0,1], \bar{h}\left(k \in a^{*}=(1,0) \mid p\right)=\bar{h}(k \in$ $\left.a^{*}=(0,1) \mid p\right)=\bar{h}\left(k \in a^{*}=(1,1) \mid p\right)$, where $\bar{h}\left(k \in a^{*}=i \mid p\right)$ is the average conditional density of $k$ when $k$ is in equilibrium set $i$.

${ }^{14}$ Formally, $\bar{h}\left(k \in a^{*}=i \mid p\right)$ has an infimum and a supremum which are in $\mathbb{R}^{++}$ for all $i \in\{(1,0),(0,1)\}$ and for all $p \in(0,1]$. To understand why this condition is sufficient consider the following example. The supremum is twice as large as the infimum. Look at Figure 1 and determine the $p$ value for which the interval of $k$ values with equilibrium $a^{*}=(1,0)$ is twice as large as the interval with $a^{*}=(0,1)$. When a $p$ is drawn which lies above this critical value we can be sure that it is more likely that the costs $k$ will lie in the interval with $a^{*}=(1,0)$ than in the interval with $a^{*}=(0,1)$. So it is more likely that $I$ invests in research than that $E$ does. One can also easily construct a lower critical value of $p$ and show that it is vice versa when $p$ is low enough.

${ }^{15}$ Think of a joint probability distribution lying over Figure 1. Ignore the density when $I$ does not invest. Then calculate $I$ 's center of mass. Make the same steps for $E$. For "many distributions", $I$ 's center is further to the right than E's.
} 


\subsection{COMPARISON TO THE LITERATURE}

In our model each firm possess one idea, which can be interpreted as a box. Each box contains either nothing or the innovation. ${ }^{16}$ To open its box a firm has to invest. ${ }^{17}$ This conception implies that the success probability of research is exogenous, and if a box turns out empty there is no way for that firm to get the innovation. Since each box can be opened at most once, the game is not repeated. ${ }^{18}$

The models differ greatly with respect to uncertainty. GN consider no uncertainty in the research process. In RE the uncertainty effectively concerns only the arrival date of the innovation, because the game is repeated unless one firm is successful. In our model, on the other hand, it is uncertain whether a firm's idea is realizable; see above. This type of uncertainty is extremely important in reality, see Freeman and Soete (1997, Ch. 8), Scotchmer (2004, pp. 40, 55), or DiMasi (2001).

In our model research is a powerful preemption device if and only if it is likely to succeed. In contrast, in GN preemption is always possible, in RE never. When preemption is possible, it is worthwhile due to the efficiency effect. Consequently, the efficiency effect is the driving force in GN, in our model when the success probability is high, and does not play a role in RE. When preemption is not possible, the efficiency effect is not important, and the replacement effect steps in. Hence it predominates in $\mathrm{RE}$, in our model when the success probability is low, and not at all in GN. Moreover, the probability that a monopoly persists is below one-half in RE, equal to one in GN, and between three-quarters and one in our model. Hence, regarding the importance of the relevant effects and the persistence of monopoly, we take a position between RE and GN.

\subsection{TIMING AND ROBUSTNESS}

We assumed that firms decide sequentially about investing in research. When instead they decide simultaneously, preemption is not possible, and

\footnotetext{
${ }^{16}$ With this interpretation nature determines success or failure already at Stage 1. Since firms do not know the realization until Stage 3 this modification of the timing does not change the model in any way.

${ }^{17}$ This description is in line with Scotchmer's (2004) statements that "[a]n innovation requires both an idea and an investment in it" (p. 39) and that "some research efforts do not pay off with certainty ... [and] failures obviously cannot be identified in advance" (p. 40).

${ }^{18}$ An interesting extension would be that firms have several boxes. However, even when one assumes that each firm can open at most one box the analysis gets cumbersome because the number of cases multiplies. Another interesting extension would be that firms can manipulate the type of their box, or that they can influence upfront what type they likely will receive.
} 
our results are no longer valid. While arbitrary from a theoretical point of view, the assumption of a sequential investment game is not unusual in the literature and also has an intuitive appeal: First, in contrast to the incumbent, the potential entrant might need some time to gather information about the market or to obtain funding. Second, Freeman and Soete (1997, p. 202) argue that "a firm which is closely in touch with the requirements of its customers may recognize potential markets". So the incumbent but not the potential entrant, may be quicker in developing new ideas. Third, with the incumbent being already prominent among market participants, its activity may be visible for everyone, while the entrepreneur's may not. Hence, only the incumbent may be able to credibly preannounce its investment decision.

In an earlier version of the paper we considered a different timing, where the potential entrant observes incumbent's success or failure before it decides about its investment. This does not change our results substantially. The same is true for the following extensions: (i) heterogenous research costs or success probabilities, (ii) patents, ${ }^{19}$ (iii) Cournot competition, (iv) product innovations, and (v) correlated success probabilities.

\section{WELFARE ANALYSIS}

In Section 3 we analyzed the positive aspects of our model. Now we explore the normative implications. In the second best world where prices cannot be regulated we seek to answer the question whether there is too much or too little investment from a welfare point of view. The literature (see Tirole 1988, p. 399) has found two counteracting effects. First, there is the nonappropriability of social surplus effect: firms may underinvest because the innovator typically does not receive the whole social surplus created by its innovation. Second, firms may overinvest due to the business stealing effect: the innovator may not take into account that it steals the rival's business.

\footnotetext{
${ }^{19}$ Our non-extended model can be interpreted in two ways: (i) There are no patents and each firm keeps details of its innovation secret so that an outsider cannot imitate. This interpretation is empirically justified because "patents are regarded as a necessary incentive for innovation in only a few industries" (Cohen 1995, p. 227). See also Scotchmer (2004, Ch. 9). (ii) There are patents but both firms innovations are different in the sense that each firm can get a patent on its technology. Additionally note, that there are patents in RE and GN is not crucial for their results: Without patents and with Bertrand competition a firm no longer wants to engage in research when its competitor was already successful.
} 
P R O P O S I T I O N 4: When the innovation is non-drastic and the incumbent preempts the potential entrant then firms may overinvest. In all other cases firms do not overinvest and may underinvest.

We first give the intuition for the case where the innovation is nondrastic and the incumbent preempts the potential entrant. When incumbent's research is successful it may set a price which is almost the same as when it would have the old technology. So the consumer surplus is hardly affected through the innovation. That is, the nonappropriability of social surplus effect is weak. Observe that through the threat of entry, the incumbent is "forced" to steal its own business. Hence the business stealing effect is powerful and may dominate. Put differently, no investment of both firms may be socially desirable.

In contrast, when the innovation is drastic, the successful incumbent sets a much lower price than it would set without the innovation. So the expected consumer surplus increases greatly and the nonappropriability effect dominates. Similar arguments apply for the case where only the potential invests.

One may presume that when both firms invest this may not be socially desirable: research effort is duplicated and so both firms may yield the innovation. This suspicion is false. When both firms are successful the consumer price is only $\underline{c}$, which results in a dominant nonappropriability effect.

Subsidies. - Suppose that the only policy instrument of a government is a research subsidy. Through subsidies the government can change firms' investment decisions, since firms determine their investments on the basis of the net costs. Proposition 4 shows that subsidies are especially relevant to support drastic innovations because for these innovations firms sometimes underinvest but never overinvest.

Targeting subsidies to potential entrants and not to incumbents has two potential advantages. First, the equilibrium $a^{*}=(0,1)$ is socially weakly preferred to $a^{*}=(1,0) \cdot{ }^{20}$ Second, promising a subsidy to $E$ in case that it invests can push $I$ to preempt $E$. Hence, the subsidy is not paid. Nonetheless a previously unexplored research project may now be investigated.

\footnotetext{
${ }^{20}$ When the innovation is non-drastic the preference is strict: total welfare is higher when there is a duopoly in which $E$ has the innovation than in a monopoly where $I$ has it.
} 


\section{EXTENSION: AUCTION SETTING}

GN's research process is commonly interpreted as a first-price auction with complete and perfect information (Reinganum, 1984). Next, we integrate such an auction setting and show that our results change substantially.

Suppose $I$ and $E$ bid for the service of a firm which implements a research project for the winner. ${ }^{21}$ The auction is held before it is clear whether the research project will be successful. ${ }^{22}$ A firm's valuation is its willingness to pay for victory, i.e., the difference in its expected profit between winning and losing.

P R O P O S I T I O N 5: The incumbent always wins if the innovation is non-drastic.

So with an auction setting and a non-drastic innovation there never is entry. This result coincides completely with GN. ${ }^{23}$ Intuitively, since $I$ can always outbid $E$, preemption is always possible. Given that the innovation is non-drastic, then, by virtue of the efficiency effect, I's valuation is strictly higher than E's, and preemption is indeed worthwhile. In contrast, when the innovation is drastic firms' valuations are the same and one has to specify a tie-breaking rule. However, if there is only a bit of uncertainty whether an innovation is indeed drastic, $I$ 's valuation is higher than $E$ 's, and so $I$ will win the auction. Hence generically, $E$ never does research, and entry never occurs. This insight is new.

\section{CONCLUSIONS}

We presented a simple model in which both, the replacement and the efficiency effect are present. We showed that research is a powerful preemption device if and only if it is likely to succeed. This results in the predominance of the efficiency effect when the success probability of research is high and the predominance of the replacement effect when it is low.

\footnotetext{
${ }^{21}$ This need not be taken literally. GN's interpretation is that the firms are in a race, and the firm which invests most wins. Another is that firms compete for scarce and essential resources, and so only the firm which invests the most gets them.

${ }^{22}$ The alternative timing is that the auction is held afterwards. Then either a project with a success probability of one or zero is auctioned. In the latter case holding an auction is superfluous. The former case is a just special case in the setting of the original timing. Hence, the alternative timing needs no separate investigation.

${ }^{23}$ We allow for an uncertain research process. GN consider uncertainty only verbally, but it is not clear to us what type of uncertainty they mean. Yi (1995) couples an auction with RE's model, and his result is that the entrant will never do research.
} 


\section{APPENDIX}

It is useful to define $\mu^{J}(p)$ as the probability that firm $J \in\{I, E\}$ will invest in research. Also define $\tau(p)\left(=p \mu^{E}(p)\right)$ as the probability that entry will occur. Both variables are measured after $p$ and before $k$ is drawn.

\subsection{PROOF OF PROPOSITION 1}

From Lemma 2 we get that

$$
\mu^{I}(p)=\int_{0}^{\left(1-\pi^{I}(\bar{c},-)\right)(1-p) p} h(k \mid p) d k+\int_{\pi^{E}(\bar{c}, \underline{c})(1-p) p}^{\pi^{E}(\bar{c}, \underline{c}) p} h(k \mid p) d k
$$

and

$$
\mu^{E}(p)=\int_{0}^{\left(1-\pi^{I}(\bar{c},-)\right)(1-p) p} h(k \mid p) d k+\int_{\left(1-\pi^{I}(\bar{c},-)\right)(1-p) p}^{\pi^{E}(\bar{c}, \underline{c})(1-p) p} h(k \mid p) d k .
$$

Hence, $\mu^{I}(p)>\mu^{E}(p)$ if and only if

$$
\int_{\pi^{E}(\bar{c}, \underline{c})(1-p) p}^{\pi^{E}(\bar{c}, \underline{c}) p} h(k \mid p) d k>\int_{\left(1-\pi^{I}(\bar{c},-)\right)(1-p) p}^{\pi^{E}(\bar{c}, \underline{c})(1-p) p} h(k \mid p) d k .
$$

So $\mu^{I}(p)>\mu^{E}(p)$ if and only if it is more likely that the replacement effect will determine the outcome than that the efficiency effect will.

Using A1, (6) is

$$
p^{2} \pi^{E}(\bar{c}, \underline{c})>(1-p) p\left[\pi^{E}(\bar{c}, \underline{c})-\left(1-\pi^{I}(\bar{c},-)\right)\right] .
$$

So $\mu^{I}(p)>\mu^{E}(p)$ if (and only if)

$$
p>\dot{p}:=\frac{\pi^{E}(\bar{c}, \underline{c})-\left(1-\pi^{I}(\bar{c},-)\right)}{2 \pi^{E}(\bar{c}, \underline{c})-\left(1-\pi^{I}(\bar{c},-)\right)} .
$$

From Lemma 1 follows that $\dot{p} \in(0,1)$. Similarly, $\mu^{I}(p)<\mu^{E}(p)$ if (and only if) $p<\dot{p}$. Note, the "only if" part is not included in Proposition 1 because it is an artefact of the uniform assumption upon $h$.

\subsection{PROOF OF PROPOSITION 2}

Start with some notation. Let the expected $p$, conditional that firm $J$ invests, be

$$
P^{J}:=\int_{0}^{1} p g^{J}(p) d p
$$


where

$$
g^{J}(p):=\frac{g(p) \mu^{J}(p)}{\int_{0}^{1} g(p) \mu^{J}(p) d p}
$$

is the density of $p$, conditional that $J \in\{I, E\}$ invests. The associated distribution function is denoted by $G^{J}(p)$. We seek to show that $P^{I}>$ $P^{E}$.

Step 1. Using Lemma 2 and A1,

$$
\frac{d\left(\frac{\mu^{I}(p)}{\mu^{E}(p)}\right)}{d p}=\frac{1}{(1-p)^{2}} .
$$

Since $\frac{g^{I}(p)}{g^{E}(p)}=\frac{\mu^{I}(p)}{\mu^{E}(p)} \frac{\int_{0}^{1} g(r) \mu^{E}(r) d r}{\int_{0}^{1} g(r) \mu^{I}(r) d r}$, it follows that $d\left(\frac{g^{I}(p)}{g^{E}(p)}\right) / d p$ is positive and finite $\forall p \in(0,1)$.

Step 2. Claim: $g^{I}(p)<g^{E}(p)$ for $p \rightarrow 0$ and $g^{I}(p)>g^{E}(p)$ for $p \rightarrow 1$.

Proof: Since $g(p)$ and $\mu^{J}(p)$ are positive and finite $\forall p \in(0,1]$, it follows that $g^{J}(p)$ is positive and finite $\forall p \in(0,1]$ as well. From step $1, d\left(\frac{g^{I}(p)}{g^{E}(p)}\right) / d p>0 \forall p \in(0,1)$, and by definition $\int_{0}^{1} g^{I}(p) d p=1$ and $\int_{0}^{1} g^{E}(p) d p=1$. Hence it must hold that $g^{I}(p)<g^{E}(p)$ for $p \rightarrow 0$, and $g^{I}(p)>g^{E}(p)$ for $p \rightarrow 1$.

Step 3. Claim: there exists a $\tilde{p} \in(0,1)$ such that $g^{I}(p)=(<,>) g^{E}(p)$ for $p=(<,>) \tilde{p}$.

ProOF: Step 1 says that $\frac{d \frac{g^{I}(p)}{g^{E}(p)}}{d p}$ is positive and finite $\forall p \in(0,1)$. So $\frac{g^{I}(p)}{g^{E}(p)}$ is continuous and increasing in $p, \forall p \in(0,1)$. This implies, together with the intermediate value theorem and step 2 , that there exists a $\tilde{p} \in(0,1)$ such that $g^{I}(\tilde{p})=g^{E}(\tilde{p})$. Since $\frac{g^{I}(p)}{g^{E}(p)}$ is increasing in $p$ $\forall p \in(0,1)$, and $g^{I}(p)<(>) g^{E}(p)$ for $p \rightarrow 0(1)$ by step 2 , it holds that $g^{I}(p)<(>) g^{E}(p)$ for $p<(>) \tilde{p}$.

Step 4. By Proposition 6.D.1 in Mas-Colell, Whinston, and Greene (1995, p. 195) $g^{I}(p)$ first-order stochastically dominates $g^{E}(p)$ if and only if

$$
\int_{0}^{x} g^{I}(p) d p \leq \int_{0}^{x} g^{E}(p) d p \quad \forall x \in(0,1] .
$$

We seek to show a slightly different property.

Claim: The inequality in $(7)$ is strict $\forall x \in(0,1)$.

Proof: Suppose there exists a $\hat{x} \in(0,1)$ such that $\int_{0}^{\hat{x}} g^{I}(p) d p \geq$ $\int_{0}^{\hat{x}} g^{E}(p) d p$. 
Case 1: $\hat{x} \leq \tilde{p}$. From step $3, g^{I}(p)<g^{E}(p) \forall p \in(0, \tilde{p})$, and $g^{I}(\tilde{p})=$ $g^{E}(\tilde{p})$. Hence, $\int_{0}^{\hat{x}} g^{I}(p) d p \geq \int_{0}^{\hat{x}} g^{E}(p) d p$ is false.

Case 2: $\hat{x}>\tilde{p}$. If $\int_{0}^{\hat{x}} g^{I}(p) d p \geq \int_{0}^{\hat{x}} g^{E}(p) d p$, then

$$
\int_{\hat{x}}^{1} g^{I}(p) d p \leq \int_{\hat{x}}^{1} g^{E}(p) d p
$$

since $\int_{0}^{1} g^{I}(p) d p=1$ and $\int_{0}^{1} g^{E}(p) d p=1$. From step $3, \forall p \in(\tilde{p}, 1)$ it is true that $g^{I}(p)>g^{E}(p)$. From Lemma 2 follows that $g^{E}(1)=0$. By definition $g^{I}(1) \geq 0$. Hence, (8) is false.

Step 5. Using the definition of $P^{E}$ and $P^{I}$, we get by integrating by parts that

$$
P^{I}=1-\int_{0}^{1} G^{I}(p) d p, P^{E}=1-\int_{0}^{1} G^{E}(p) d p .
$$

From step 4 we know that $G^{I}(p)<G^{E}(p) \forall p \in(0,1)$. By definition $G^{I}(1)=G^{E}(1)$. Hence, $\int_{0}^{1} G^{I}(p) d p<\int_{0}^{1} G^{E}(p) d p$ and so $P^{I}>P^{E}$.

\subsection{PROOF OF PROPOSITION 3}

A1 implies that $h(k \mid p)=h(p) \forall k \in \mathcal{S}$. By the definition of a density,

$$
\int_{0}^{p \pi^{E}(\bar{c}, \underline{c})} h(p) d k \leq 1
$$

or rewritten

$$
h(p) \leq \frac{1}{p \pi^{E}(\bar{c}, \underline{c})} .
$$

Together with Lemma 2 this implies that

$$
\mu^{E}(p)=h(p) \pi^{E}(\bar{c}, \underline{c})(1-p) p \leq \frac{1}{p \pi^{E}(\bar{c}, \underline{c})} \pi^{E}(\bar{c}, \underline{c})(1-p) p=1-p .
$$

Since, by definition $\tau(p)=p \mu^{E}(p)$, it follows that $\tau(p) \leq p(1-p)$.

\subsection{PROOF OF PROPOSITION 4}

The total welfare, excluding potential investments in research, when the incumbent has production costs $c^{I}$ and the potential entrant costs $c^{E}$ is denoted by $t\left(c^{I}, c^{E}\right)$. It consists of the firms' Bertrand profits and consumer welfare (for sake of clearness we do not use the normalization 
$\left.\pi^{I}(\underline{c},-)=1\right)$

$$
\begin{aligned}
t(\bar{c},-) & =\int_{\phi^{I}(\bar{c},-)}^{\infty} D(\phi) d \phi+\pi^{I}(\bar{c},-), \\
t(\underline{c},-) & =\int_{\phi^{I}(\underline{c},-)}^{\infty} D(\phi) d \phi+\pi^{I}(\underline{c},-), \\
t(\underline{c}, \underline{c}) & =\int_{\underline{c}}^{\infty} D(\phi) d \phi+0, \\
t(\bar{c}, \underline{c}) & =\int_{\phi^{E}(\bar{c}, \underline{c})}^{\infty} D(\phi) d \phi+\pi^{E}(\bar{c}, \underline{c}),
\end{aligned}
$$

where $\phi^{J}\left(c^{I}, c^{E}\right)$ is the price set by firm $J \in\{I, E\}$. Let $T\left(a^{I}, a^{E}\right)$ be the expected total welfare taking into account firms' investments. Straightforward calculations yield:

$$
\begin{aligned}
T(0,0)= & t(\bar{c},-), \\
T(0,1)= & p t(\bar{c}, \underline{c})+(1-p) t(\bar{c},-)-k, \\
T(1,0)= & p t(\underline{c},-)+(1-p) t(\bar{c},-)-k, \\
T(1,1)= & p^{2} t(\underline{c}, \underline{c})+p(1-p) t(\underline{c},-) \\
& +p(1-p) t(\bar{c}, \underline{c})+(1-p)^{2} t(\bar{c},-)-2 k .
\end{aligned}
$$

Observe that for a drastic innovation $\phi^{E}(\bar{c}, \underline{c})=\phi^{I}(\underline{c},-)$ and so $T(0,1)=$ $T(1,0)$. However, when the innovation is non-drastic $\phi^{E}(\bar{c}, \underline{c})<\phi^{I}(\underline{c},-)$ and so $T(0,1)>T(1,0)$. Next we explore the question whether there is socially too much or too little investment. We look at the second best world in which pricing cannot be regulated.

Overinvestment when $a^{*}=(1,0)$ ? - Consider first the case that in equilibrium only the incumbent invests. Is it socially desirable that no firm invests? Using the formulas derived before yields

$$
T(1,0)-T(0,0)=p\left[\int_{\phi^{I}(\underline{c},-)}^{\phi^{I}(\bar{c},-)} D(\phi) d \phi+\pi^{I}(\underline{c},-)-\pi^{I}(\bar{c},-)\right]-k .
$$

From Lemma 2 we know that for $a^{*}=(1,0)$,

$$
k=p \pi^{E}(\bar{c}, \underline{c})-(+ \text { term }),
$$


where the positive term can be arbitrarily small. Then

$$
\begin{aligned}
& T(1,0)-T(0,0)= \\
& \quad p\left[\int_{\phi^{I}(\underline{c},-)}^{\phi^{I}(\bar{c},-)} D(\phi) d \phi+\pi^{I}(\underline{c},-)-\pi^{I}(\bar{c},-)-\pi^{E}(\bar{c}, \underline{c})\right]+(+ \text { term }) .
\end{aligned}
$$

For a drastic innovation, $\pi^{I}(\underline{c},-)=\pi^{E}(\bar{c}, \underline{c})$. The profit term $\pi^{I}(\bar{c},-)$ can be rewritten as

$$
\int_{\bar{c}}^{\phi^{I}(\bar{c},-)} D\left(\phi^{I}(\bar{c},-)\right) d \phi .
$$

Since we assumed that the monopolist' optimal price is unique we have that for a drastic innovation $\phi^{I}(\underline{c},-) \leq \bar{c}$. Hence, $T(1,0)-T(0,0)>0$.

However, when the innovation is non-drastic, then it can be the case that $T(1,0)-T(0,0)<0$. This is true in the following example: $(+$ term $)$ is small; $\phi^{I}(\bar{c},-) \approx \phi^{I}(\underline{c},-)$ so that the integral term in (9) is small; let $\pi^{I}(\underline{c},-)-\pi^{I}(\bar{c},-)$ be well below $\pi^{E}(\bar{c}, \underline{c})$ (this is possible due to the replacement effect).

Overinvestment when $a^{*}=(0,1)$ ? — When in equilibrium only $E$ invests, is it socially desirable that no firm invests?

$$
T(0,1)-T(0,0)=p\left[\int_{\phi^{E}(\bar{c}, \underline{c})}^{\phi^{I}(\bar{c},-)} D(\phi) d \phi+\pi^{E}(\bar{c}, \underline{c})-\pi^{I}(\bar{c},-)\right]-k .
$$

From Lemma 2, when $a^{*}=(0,1)$ then

$$
k=\pi^{E}(\bar{c}, \underline{c})(1-p) p-(+ \text { term }),
$$

and so

$$
T(0,1)-T(0,0)=p\left[\int_{\phi^{E}(\bar{c}, \underline{c})}^{\phi^{I}(\bar{c},-)} D(\phi) d \phi+p \pi^{E}(\bar{c}, \underline{c})-\pi^{I}(\bar{c},-)\right]+(+ \text { term }) .
$$

Are the terms in the square brackets also positive if $p \rightarrow 0$ ? Yes, since $\phi^{I}(\bar{c},-)>\bar{c} \geq \phi^{E}(\bar{c}, \underline{c})$ and the demand is decreasing in the price:

$\int_{\phi^{E}(\bar{c}, \underline{c})}^{\phi^{I}(\bar{c},-)} D(\phi) d \phi \geq \int_{\bar{c}}^{\phi^{I}(\bar{c},-)} D(\phi) d \phi>\int_{\bar{c}}^{\phi^{I}(\bar{c},-)} D\left(\phi^{I}(\bar{c},-)\right) d \phi=\pi^{I}(\bar{c},-)$.

Obviously, for all $p$ the terms in the square brackets are positive, and so it holds that $T(0,1)-T(0,0)>0$.

Overinvestment when $a^{*}=(1,1)$ ? — Next consider the case when 
both firms invest in research. Is it socially desirable that instead only the potential entrant invests?

$$
\begin{aligned}
& T(1,1)-T(0,1) \\
& =p\left[p\left(\int_{\underline{c}}^{\phi^{E}(\bar{c}, \underline{c})} D(\phi) d \phi-\pi^{E}(\bar{c}, \underline{c})\right)+\right. \\
& \left.(1-p)\left(\int_{\phi^{I}(\underline{c},-)}^{\phi^{I}(\bar{c},-)} D(\phi) d \phi+\pi^{I}(\underline{c},-)-\pi^{I}(\bar{c},-)\right)\right]-k .
\end{aligned}
$$

From Lemma 2 we know that when $a^{*}=(1,1)$, then

$$
k=p(1-p)\left(\pi^{I}(\underline{c},-)-\pi^{I}(\bar{c},-)\right)-(+ \text { term }) .
$$

Using this we get that

$$
\begin{aligned}
T(1,1)-T(0,1)=p & {\left[p\left(\int_{\underline{c}}^{\phi^{E}(\bar{c}, \underline{c})} D(\phi) d \phi-\pi^{E}(\bar{c}, \underline{c})\right)\right.} \\
& \left.+(1-p)\left(\int_{\phi^{I}(\underline{c},-)}^{\phi^{I}(\bar{c},-)} D(\phi) d \phi\right)\right]+(+ \text { term }) .
\end{aligned}
$$

The second integral is nonnegative because $\phi^{I}(\underline{c},-) \leq \phi^{I}(\bar{c},-)$, see Tirole (1988, p. 66). The term $\int_{\underline{c}}^{\phi^{E}(\bar{c}, \underline{c})} D(\phi) d \phi-\pi^{E}(\bar{c}, \underline{c})$ can be rewritten as

$$
\int_{\underline{c}}^{\phi^{E}(\bar{c}, \underline{c})} D(\phi) d \phi-\int_{\underline{c}}^{\phi^{E}(\bar{c}, \underline{c})} D\left(\phi^{E}(\bar{c}, \underline{c})\right) d \phi
$$

which is positive because demand is decreasing in the price and $\phi^{E}(\bar{c}, \underline{c})>$ c. Hence, $T(1,1)-T(0,1)>0$.

We know that $T(0,1) \geq T(1,0)$. Hence also $T(1,1)-T(1,0)>0$.

Finally, is $T(1,1)$ socially preferred to $T(0,0)$ ?

$$
\begin{aligned}
T(1,1)-T(0,0)=p^{2}[t(\underline{c}, \underline{c})- & t(\underline{c},-)-t(\bar{c}, \underline{c})+t(\bar{c},-)] \\
& +p[t(\underline{c},-)+t(\bar{c}, \underline{c})-2 t(\bar{c},-)]-2 k .
\end{aligned}
$$

Again, for $a^{*}=(1,1)$ it holds that

$$
k=p(1-p)\left(\pi^{I}(\underline{c},-)-\pi^{I}(\bar{c},-)\right)+(+ \text { term }) .
$$


Hence, after some calculations,

$$
\begin{aligned}
& T(1,1)-T(0,0) \\
= & p^{2}\left[\int_{\underline{c}}^{\phi^{E}(\bar{c}, \underline{c})} D(\phi) d \phi-\int_{\phi^{I}(\underline{c},-)}^{\phi^{I}(\bar{c},-)} D(\phi) d \phi-\pi^{E}(\bar{c}, \underline{c})+\pi^{I}(\underline{c},-)-\pi^{I}(\bar{c},-)\right] \\
+ & p\left[\int_{\phi^{I}(\underline{c},-)}^{\phi^{I}(\bar{c},-)} D(\phi) d \phi+\int_{\phi^{E}(\bar{c}, \underline{c})}^{\phi^{I}(\bar{c},-)} D(\phi) d \phi+\pi^{E}(\bar{c}, \underline{c})-\pi^{I}(\underline{c},-)\right]+(+ \text { term }) .
\end{aligned}
$$

If $p=1$, one gets that

$$
T(1,1)-T(0,0)=\int_{\underline{c}}^{\phi^{I}(\bar{c},-)} D(\phi) d \phi-\pi^{I}(\bar{c},-)+(+ \text { term }),
$$

which is obviously positive.

If $p \rightarrow 0$, only the last line of (10) is important. The replacement effect holds and so $\pi^{E}(\bar{c}, \underline{c})-\pi^{I}(\underline{c},-)=-\pi^{I}(\bar{c},-)+(+$ term $)$, see Lemma 1. So the last line of (10) can be rewritten as

$$
p\left[\int_{\phi^{I}(\underline{c},-)}^{\phi^{I}(\bar{c},-)} D(\phi) d \phi+\int_{\phi^{E}(\bar{c}, \underline{c})}^{\phi^{I}(\bar{c},-)} D(\phi) d \phi-\pi^{I}(\bar{c},-)\right]+(+ \text { term }) .
$$

From before (see the case $a^{*}=(0,1)$ ) we know that $\int_{\phi^{E}(\bar{c}, \underline{c})}^{\phi^{I}(\bar{c}-)} D(\phi) d \phi>$ $\pi^{I}(\bar{c},-)$, and so the last line is positive. Hence, also for $p \rightarrow 0, T(1,1)-$ $T(0,0)>0$. Since for $p=1$ and for $p \rightarrow 0$ we have $T(1,1)-T(0,0)>0$, and through the functional form of $(10)$, we get that $T(1,1)-T(0,0)>0$ $\forall p \in(0,1]$.

Underinvestment. - Note, when $a^{*}=(0,0)$ the previous analysis of the case $a^{*}=(1,0)$ applies expect that there is no positive term, but instead a negative term. When $k$ is close to the boundary where $a^{*}=(1,0)$, the negative term is close to zero. Hence, our analysis shows that it may be socially desirable that one firm invests when in fact no firm invests. The same arguments hold when in fact one firm invests and one asks the question whether investments of both firms is socially preferable. Hence, firm may invest too little.

\subsection{PROOF OF PROPOSITION 5}

Denote firm $J$ 's valuation as $v^{J}, J \in\{I, E\}$. I's valuation $v^{I}$ is given through the $k$ which equates (5), and so $v^{I}=p$. Similarly, from (1) we get $v^{E}=\pi^{E}(\bar{c}, \underline{c}) p$. If the innovation is non-drastic, $\pi^{E}(\bar{c}, \underline{c})<1$, and 
hence $v^{I}>v^{E} \forall p \in(0,1]$. Since we consider a first-price auction with complete and perfect information, $I$ will always win.

\section{LITERATURE}

Arrow, Kenneth (1962). Economic Welfare and the Allocation of Resources for Inventions. In The Rate and Direction of Inventive Activity, ed. R. Nelson. NJ: Princeton University Press, 609-624.

Astebo, Thomas (2003). The Return to Independent Invention: Evidence of Unrealistic Optimism, Risk Seeking or Skewness Loving? Economic Journal 113, 226-239.

Baumol, William (2004). Education for Innovation: Entrepreneurial Breakthroughs vs. Corporate Incremental Improvements. NBER Working Paper 10578.

Beath, John, Yannis Katsoulacos, and David Ulph (1989a). The Game-Theoretic Analysis of Innovation: A Survey. Bulletin of Economic Research 41, 163-184.

Beath, John, Yannis Katsoulacos, and David Ulph (1989b). Strategic R\&D Policy. Economic Journal 99, 74-83.

Bianchi, Milo and Magnus Henrekson (2005). Is Neoclassical Economics still Entrepreneurless? Kyklos 58(3), 353-377.

Blume, Andreas (2003). Bertrand with fudge. Economic Letters 78, 167-168.

Cohen, Wesley (1995). Empirical Studies of Innovative Activity. In Stoneman, Paul (Ed.), Handbook of the Economics of Innovation and Technological Change, Oxford: Blackwell Publishers, 182-264.

De Meza, David and Clive Southey (1996). The Borrower's Curse: Optimism, Finance and Entrepreneurship. Economic Journal 106(435), 375-386.

Denicolo, Vincenzo (2001). Growth with non-drastic innovations and the persistence of leadership. European Economic Review 45, 1399-1413.

DiMasi, Joseph A. (2001). Risks in new drug development: Approval success rates for investigational drugs. Clinical Pharmacology and Therapeutics 69(5), 297-307.

Dixit, Avinash (1980). The Role of Investment in Entry-Deterrence. Economic Journal 90(357), 95-106.

Economist, The (2007). Something new under the sun. A special report on innovation, 13th October 2007, 3-4.

Etro, Federico (2004). Innovation by Leaders. Economic Journal 114, 281-303.

Freeman, Chris and Luc Soete (1997). The Economics of Industrial Innovation. Third edition. Cambridge: MIT Press.

Fudenberg, Drew and Jean Tirole (1986). Dynamic Models of Oligopoly. Volume 3 of Fundamentals of Pure and Applied Economics, ed. Lesourne and Sonnenschein. London: Harwood.

Geroski, Paul (1995). What do we know about entry? International Journal of Industrial Organization 13, 421-440.

Gilbert, Richard J. (2006). Looking for Mr. Schumpeter: Where Are We in the Competition-Innovation Debate? In Innovation Policy and the Economy, ed. Jaffe, Adam B., Lerner, Josh and Stern, Scott. Cambridge: MIT Press, 159-215.

Gilbert, Richard J. and David M. G. Newbery (1982). Preemptive Patenting and the Persistence of Monopoly. American Economic Review 72, 514-526. 
Gilbert, Richard J. and David M. G. Newbery (1984). Uncertain Innovation and the Persistence of Monopoly: Comment. American Economic Review 74(1), 238-242.

Hermalin, Benjamin (2009). Lecture Notes for Economics. http://faculty.haas.berkeley. edu/hermalin/LectureNotes201b_v5.pdf.

Kihlstrom, Richard E. and Jean-Jacques Laffont (1979). A General Equilibrium Entrepreneurial Theory of Firm Formation Based on Risk Aversion. Journal of Political Economy 87(4), 719-748.

Mansfield, Edwin, J. Rapaport, A. Romeo, E. Villani, S. Wagner, and F. Husic (1977). The production and application of new industrial technology. New York: Norton.

Mas-Colell, Andreu, Michael D. Whinston, and Jerry R. Greene (1995). Microeconomic Theory. Oxford: Oxford University Press.

Reinganum, Jennifer (1983). Uncertain Innovation and the Persistence of Monopoly. American Economic Review 73(4), 741-748.

Reinganum, Jennifer (1984). Uncertain Innovation and the Persistence of Monopoly: Reply. American Economic Review 74(1), 243-246.

Rosen, Richard J. (1991). Research and Development with Asymmetric Firm Sizes. RAND Journal of Economics 22(3), 411-429.

Scherer, Frederic M. and David Ross (1990). Industrial Market Structure and Economic Performance. Third edition. Boston: Houghton Mifflin Company.

Scotchmer, Suzanne (2004). Innovation and Incentives. Cambridge: MIT Press.

Spence, A. Michael (1977). Entry, Capacity, Investment and Oligopolistic Pricing. Bell Journal of Economics 8(2), 534-544.

Tirole, Jean (1988). The Theory of Industrial Organization. Cambridge: MIT Press. Yi, Sang-Seung (1995). Uncertain innovation and persistence of monopoly revisited. Economics Letters 49, 319-322. 